\title{
Mellin's Transform and Application to Some Time Series Models
}

\author{
S. S. Appadoo, ${ }^{1}$ A. Thavaneswaran, ${ }^{2}$ and S. Mandal $^{2}$ \\ ${ }^{1}$ Department of Supply Chain Management, University of Manitoba, Winnipeg, MB, Canada R3T 2N2 \\ ${ }^{2}$ Department of Statistics, University of Manitoba, Winnipeg, MB, Canada R3T 2N2
}

Correspondence should be addressed to S. S. Appadoo; appadoo@cc.umanitoba.ca

Received 8 July 2013; Accepted 26 August 2013; Published 18 February 2014

Academic Editors: F. Ding and D. Luchinsky

Copyright (C) 2014 S. S. Appadoo et al. This is an open access article distributed under the Creative Commons Attribution License, which permits unrestricted use, distribution, and reproduction in any medium, provided the original work is properly cited.

This paper uses the Mellin transform to establish the means, variances, skewness, and kurtosis of fuzzy numbers and applied them to the random coefficient autoregressive (RCA) time series models. We also give a close form expression to the moment generating function related to fuzzy numbers. It is shown that the results of the proposed time series models are consistent with those of the conventional time series models and that the developed concepts are straightforward and easily implemented.

\section{Introduction}

The use of fuzzy set theory as a methodology for modeling and analyzing certain financial problems is of particular interest to a number of researchers due to fuzzy set theory's ability to quantitatively and qualitatively model those problems which involve vagueness and imprecision. Fuzzy time series models provide a new avenue to deal with subjectivity observed in most financial time series models. Most of the fuzzy financial models developed so far have, generally, been confined to modeling parameters through some form of defuzzification or linear type of fuzzy numbers such as Trapezoidal Fuzzy Number (Tr.F.N.) or Triangular Fuzzy Number (T.F.N.).

There are some connections between fuzzy numbers based on convolution principle and some integral transformations such as the Mellin transform. The Mellin transform and its inverse are related to the two-sided Laplace transform. The Mellin transform is used often in applied sciences such as physics, engineering, and computer science because of its scale invariance property. In fact, this scale invariance property is analogous to the Fourier transform's shift invariance property. In particular, this transform is used for the analysis of linear time-invariant systems. This transform is considered as a transformation from the time-domain in which inputs and outputs are functions of time. Thus, the Mellin transform has significant applications in probability theory, Markov chains, renewal theory, and time series. The objective of this paper is to use the Mellin transform to establish the means, variances, skewness, and kurtosis of fuzzy numbers and then to apply to the random coefficient autoregressive (RCA) time series models. We also provide a close form expression to the moment generating function related to fuzzy numbers.

We summarize the preliminaries and notations in Section 1 . The remainder of the paper is organized as follows. Section 2 introduces the Mellin transform to obtain statistical moments of any order. The useful Mellin transforms for fuzzy numbers are derived in Section 3. We also define the moment generating function of fuzzy numbers and provide some illustrative examples. In Section 4, we discussed the fuzzy RCA model under different innovation terms. Finally, concluding remarks are given in Section 5.

1.1. Preliminaries and Notation. The purpose of this section is to recall some concepts which will be used throughout the rest of the paper. We quote definitions and properties of fuzzy sets theory with relevant operations. Most of these related definitions and properties may be found in the following two references $[1,2]$. In the sequel we will denote the family of fuzzy numbers by $\mathfrak{F}$. For any $A \in \mathfrak{F}$, we use the notation $A(\alpha)=\left[a_{1}(\alpha), a_{2}(\alpha)\right]$ for $\alpha$-level sets of $A$. 
Definition 1. Fuzzy set $A$ in $X \subset \mathfrak{R}$, the set of real numbers, is a set of ordered pairs $A=\{(x, \mu(x): x \in X\}$, where $\mu(x)$ is the membership function or grade of membership, or degree of compatibility or degree of truth of $x \in X$ which maps $x \in X$ on the real interval $[0,1]$.

Definition 2. If $\operatorname{Sup} \mu(x)=1, x \in \mathfrak{R}$, then the fuzzy set $A$ is called a normal fuzzy set in $\mathfrak{R}$.

Definition 3. The crisp set of elements that belong to the fuzzy set $A$ at least to the degree $\alpha$ is called the $\alpha$-level set (or $\alpha$ cut); that is, $A(\alpha)=\left\{x \in X \mid \mu(x) \geq \alpha, \alpha \in \mathfrak{R}^{+}\right\}$. If the set $A^{\prime}(\alpha)=\left\{x \in X \mid \mu(x)>\alpha, \alpha \in \Re^{+}\right\}$, then $A^{\prime}(\alpha)$ is called strong $\alpha$-level set (or strong $\alpha$-cut).

Definition 4. A fuzzy set $A$ is said to be a convex set if $\mu\left(\lambda x_{1}+\right.$ $\left.(1-\lambda) x_{2}\right) \geq \min \left(\mu\left(x_{1}\right), \mu\left(x_{2}\right)\right), x_{1}, x_{2} \in X$ and $\lambda \in[0,1]$. Alternatively, a fuzzy set $A$ is convex if its every $\alpha$-level sets is a convex set.

Definition 5. A fuzzy set $A$, which is both convex and normal, is defined to be a fuzzy number on the universal set $\mathfrak{R}$.

Definition 6. The standard Gaussian fuzzy number can be represented by $A=(x: \mu, \sigma, \alpha)$ with membership function as

$$
\mu_{A}(x)=\exp \left[-\frac{1}{2}\left(\frac{x-\mu}{\sigma}\right)^{2}\right]
$$

where $\mu$ is called the mean and $\sigma$ the standard deviation. Alternatively, defining the interval of confidence at level $\alpha$ as $A(\alpha)=\left[a_{1}(\alpha), a_{2}(\alpha)\right]$, and setting $\exp \left[-(1 / 2)((x-\mu) / \sigma)^{2}\right]=$ $\alpha$, we get $a_{1}(\alpha)=\mu-\sigma \sqrt{(-2 \ln \alpha)}$ and $a_{2}(\alpha)=\mu+$ $\sigma \sqrt{(-2 \ln \alpha)}$. Thus, we get the $\alpha$-cut representation of the standard gaussian fuzzy number as

$$
\begin{array}{r}
A(\alpha)=\left[a_{1}(\alpha), a_{2}(\alpha)\right] \\
=[\mu-\sigma \sqrt{(-2 \ln \alpha)}, \mu+\sigma \sqrt{(-2 \ln \alpha)}], \\
\forall, \alpha \in(0,1] .
\end{array}
$$

Definition 7. A Triangular Fuzzy Number (T.F.N.) can be represented completely by a triplet $A=\left(a_{1}, a_{2}, a_{3}\right)$, where $a_{1}<a_{2}<a_{3} \in \mathfrak{R}$ with membership function $\mu(x)$ as

$$
\mu(x)= \begin{cases}0, & x \leq a_{1}, \\ \frac{x-a_{1}}{a_{2}-a_{1}}, & a_{1} \leq x \leq a_{2}, \\ \frac{x-a_{3}}{a_{2}-a_{3}}, & a_{2} \leq x \leq a_{3}, \\ 0, & x \geq a_{3} .\end{cases}
$$

Alternatively, defining the interval of confidence at level $\alpha$ as $A(\alpha)=\left[a_{1}(\alpha), a_{2}(\alpha)\right]$ and setting $\left(x-a_{1}\right) /\left(a_{2}-a_{1}\right)=\alpha$ and $\left(x-a_{3}\right) /\left(a_{2}-a_{3}\right)=\alpha$, we get $a_{1}(\alpha)=a_{1}+\alpha\left(a_{2}-a_{1}\right)$ and $a_{2}(\alpha)$ $=a_{3}+\alpha\left(a_{2}-a_{3}\right)$. Thus, we get the parametric representation of the T.F.N. $\left[a_{1}, a_{2}, a_{3}\right]$, where $a_{1}<a_{2}<a_{3} \in X$, as

$$
\begin{array}{r}
A(\alpha)=\left[a_{1}(\alpha), a_{2}(\alpha)\right] \\
=\left[a_{1}+\alpha\left(a_{2}-a_{1}\right), a_{3}+\alpha\left(a_{2}-a_{3}\right)\right], \\
\forall, \alpha \in(0,1] .
\end{array}
$$

Definition 8. A Trapezoidal Fuzzy Number (Tr.F.N.) is represented completely by a quadruplet $A=\left(a_{1}, a_{2}, a_{3}, a_{4}\right)$, where $a_{1}<a_{2}<a_{3}<a_{4} \in X$ with membership function $\mu(x)$ given by

$$
\mu(x)= \begin{cases}0, & x \leq a_{1}, \\ \frac{x-a_{1}}{a_{2}-a_{1}}, & a_{1} \leq x \leq a_{2}, \\ 1, & a_{2} \leq x \leq a_{3}, \\ \frac{x-a_{4}}{a_{3}-a_{4}}, & a_{3} \leq x \leq a_{4}, \\ 0, & x \geq a_{4} .\end{cases}
$$

As in Definition 1, we obtain the parametric representation of the Tr.F.N. $A=\left(a_{1}, a_{2}, a_{3}, a_{4}\right)$, where $a_{1}<a_{2}<a_{3}<a_{4} \in X$, as

$$
\begin{aligned}
& A(\alpha)=\left[a_{1}(\alpha), a_{2}(\alpha)\right] \\
&=\left[a_{1}+\alpha\left(a_{2}-a_{1}\right), a_{4}+\alpha\left(a_{3}-a_{4}\right)\right], \\
& \forall \alpha \in(0,1] .
\end{aligned}
$$

In Section 2, we discuss the use of the Mellin transform to find moments of fuzzy numbers, and nonzero weighted central possibilistic moments.

\section{Probability Density Function from Membership Function}

In order to add fuzziness to data, Kaufmann and Gupta [2] obtained a membership function from a probability density function by way of a simple linear transformation, which indicates a possible conversion of a membership function into a density function. The conversion of a membership function into a probability density function can be made by one of two linear transformations, which will be elaborated in the next Section. The conversion of the membership function of a fuzzy number $\mu(x)$, into a probability density function can be achieved by using one of the two linear transformations presented in Sheen [3].

(a) Proportional probability density function (ppdf),

$$
p(x)=h_{p} \mu_{A}(x) \text {, }
$$

(b) Uniform probability density function (updf):

$$
u(x)=\mu_{A}(z)+\left(h_{u}-1\right),
$$


where $h_{p}$ and $h_{u}$ are the conversion constants which ensure that the area under the continuous probability function is equals to one. Sheen [3] shows, that for the proportional conversion, the domain of the triangular ppdf is the same as the converted T.F.N., while its height changes from 1 to $h_{p}=2 /\left(a_{3}-a_{1}\right)$. In the case of the updf function, the height of the triangular updf is given by $h_{u}=\sqrt{2 /\left(a_{3}-a_{1}\right)}$, while its vertexes are expressed as: $a_{1}^{\prime}=a_{2}-h_{u}\left(a_{2}-a_{1}\right), a_{2}^{\prime}=a_{2}$, $a_{3}^{\prime}=a_{2}+h_{u}\left(a_{3}-a_{2}\right)$. For a Tr.F.N., the height of the trapezoidal ppdf is given by $h_{p}=2 /\left[\left(a_{4}+a_{3}\right)-\left(a_{2}+a_{1}\right)\right]$. Meanwhile, the height and the vertexes, respectively, of the trapezoidal updf are expressed as:

$$
h_{u}=\frac{\left(\left(a_{2}-a_{3}\right)+\sqrt{\left(a_{2}-a_{3}\right)^{2}+2\left(a_{4}-a_{3}+a_{2}-a_{1}\right)}\right)}{\left(a_{4}-a_{3}+a_{2}-a_{1}\right)}
$$

$a_{1}^{\prime}=a_{2}-h_{u}\left(a_{2}-a_{1}\right), a_{2}^{\prime}=a_{2}, a_{3}^{\prime}=a_{3}, a_{4}^{\prime}=a_{3}+h_{u}\left(a_{4}-a_{3}\right)$.

It is found that the conversion of a fuzzy number into its corresponding proportional distribution is computationally straightforward.

2.1. The Mellin Transform and Ranking of Fuzzy Numbers. The Mellin transform $M_{x}(s)$ of a function $f(x)$, where $x$ is positive, is defined in Sheen [3] as follows:

$$
M_{x}(s)=\int_{0}^{\infty} x^{s-1} f(x) d x, \quad 0 \leq x \leq \infty
$$

The Mellin transform has a unique one-to-one correspondence; that is, $f(x) \leftrightarrow M_{x}(s)$.

The function $f(x)$ is called the inverse the Mellin transformation of $M_{x}(s)$. Under certain conditions, inverse Mellin transformation may be represented as an integral. The tables of both the Mellin transforms and inverse Mellin transforms are given by Erdelyi [4]. The Mellin transforms are virtually two-sided Laplace transforms and can be expressed either as exponential Fourier transforms or as combinations of Laplace transforms. For this reason, the Mellin transforms are found in several works on Fourier and Laplace transforms. The two most important sources are Doetsch [5] and Titchmarsh [6]. For more application on the Mellin transform, we can refer to González-Gaxiola and Santiago [7] and Frontczak and Schöbel [8] for details.

The moments of a distribution represent the expected values of the power of a random variable with a $f(x)$ distribution. In other words, the Mellin transform $M_{x}(s)=$ $E\left[X^{s-1}\right]$ provides an alternative method to establish a series of moments of a distribution if $f(x)$ is viewed as a probability density function. Comparing the first two moments of a distribution with the Mellin transform allows the mean, variance, and other moments to be expressed as follows; that is,

$$
\begin{gathered}
\mu=E[x]=M_{x}(2) \\
m_{2}=\sigma^{2}=\operatorname{Var}(X)=M_{x}(3)-\left(M_{x}(2)\right)^{2} .
\end{gathered}
$$

Thus,

$$
\begin{aligned}
& \operatorname{Var}(x)=M_{x}(3)-\left(M_{x}(2)\right)^{2}, \\
& E(x-\mu)^{3}=M_{x}(4)-3 M_{x}(2) M_{x}(3)+2\left(M_{x}(2)\right)^{3}, \\
& E(x-\mu)^{4}=M_{x}(5)-4 M_{x}(2) M_{x}(4) \\
& +6\left(M_{x}(2)\right)^{2} M_{x}(3)-3\left(M_{x}(2)\right)^{4}, \\
& \text { Skewness }(x)=\frac{E(x-\mu)^{3}}{(\sqrt{\operatorname{Var}(x)})^{3}} \\
& =\frac{M_{x}(4)-3 M_{x}(2) M_{x}(3)+2\left(M_{x}(2)\right)^{3}}{\left(\sqrt{M_{x}(3)-\left(M_{x}(2)\right)^{2}}\right)^{3}}, \\
& \operatorname{Kurtosis}(x)=\frac{E(x-\mu)^{4}}{(\operatorname{Var}(x))^{2}} \\
& =\left(M_{x}(5)-4 M_{x}(2) M_{x}(4)\right. \\
& \left.+6\left(M_{x}(2)\right)^{2} M_{x}(3)-3\left(M_{x}(2)\right)^{4}\right) \\
& \times\left(\left(M_{x}(3)-\left(M_{x}(2)\right)^{2}\right)^{2}\right)^{-1}
\end{aligned}
$$

\section{Moments Properties of Some of Fuzzy Numbers}

In this Section, on the line of Sheen [3], we discuss moment of a T.F.N fuzzy numbers and derive expression for the kurtosis and skewness.

Theorem 9. For a T.F.N., A one has the following easy to prove results. In what follows, one will assume that $\mu_{1}(x)=((x-$ $\left.\left.a_{1}\right) /\left(a_{2}-a_{1}\right)\right), \mu_{2}(x)=\left(\left(x-a_{3}\right) /\left(a_{2}-a_{3}\right)\right)$ and $h_{p}=\left(2 /\left(a_{3}-\right.\right.$ $\left.\left.a_{1}\right)\right)$. Then, we have the following results:

$$
\begin{gathered}
h_{p}=\frac{2}{a_{3}-a_{1}}, \\
M_{x}(s)=\left(\frac{h_{p}}{s(1+s)}\right) \\
\times\left(\left(\frac{a_{2}^{s} a_{1}(1+s)-\left(a_{2}^{1+s} s+a_{1}^{1+s}\right)}{\left(a_{1}-a_{2}\right)}\right)\right. \\
\left.+\left(\frac{a_{2}^{s} a_{3}(1+s)-\left(a_{3}^{1+s}+a_{2}^{1+s} s\right)}{\left(a_{2}-a_{3}\right)}\right)\right),
\end{gathered}
$$




$$
\begin{gathered}
M_{x}(2)=\frac{\left(a_{1}+a_{2}+a_{3}\right)}{3}, \\
\operatorname{Var}(x)=\frac{\left(a_{1}^{2}+a_{2}^{2}+a_{3}^{2}-a_{2} a_{1}-a_{3} a_{2}-a_{3} a_{1}\right)}{18},
\end{gathered}
$$$$
\text { Kurtosis }(x)=\frac{12}{5} \text {, }
$$

Skewness $(x)$

$$
\begin{aligned}
=( & \left(4\left(a_{1}^{3}+a_{2}^{3}+a_{3}^{3}\right)\right. \\
& -6\left(a_{1}^{2} a_{3}+a_{1}^{2} a_{2}+a_{1} a_{2}^{2}+a_{1} a_{3}^{2}+a_{3}^{2} a_{2}+a_{3} a_{2}^{2}\right) \\
& \left.+24 a_{1} a_{3} a_{2}\right) \\
\times & \left(5\left(a_{1}^{2}+a_{2}^{2}+a_{3}^{2}-a_{2} a_{1}-a_{3} a_{2}-a_{3} a_{1}\right)\right. \\
& \left.\left.\times \sqrt{\left(2 a_{1}^{2}+2 a_{2}^{2}+2 a_{3}^{2}-2 a_{2} a_{1}-2 a_{3} a_{2}-2 a_{3} a_{1}\right)}\right)^{-1}\right) .
\end{aligned}
$$

Proof. In order to find $h_{p}$, the conversion constants which ensure that the area under the continuous probability function is equal to one, we proceed as follows: set

$$
\begin{aligned}
& h_{p}\left(\int_{a_{1}}^{a_{2}}\left(\frac{x-a_{1}}{a_{2}-a_{1}}\right) d x+\int_{a_{2}}^{a_{3}}\left(\frac{x-a_{3}}{a_{2}-a_{3}}\right) d x\right)=1 \\
& h_{p}\left(\frac{(1 / 2)\left(a_{2}-a_{3}\right)\left(-a_{3}+a_{1}\right)\left(-a_{2}+a_{1}\right)}{\left(a_{2}-a_{1}\right)\left(a_{2}-a_{3}\right)}\right)=1
\end{aligned}
$$

solving for $h_{p}$; we have

$$
h_{p}=\frac{1}{\left((1 / 2) a_{3}-(1 / 2) a_{1}\right)}=\frac{2}{a_{3}-a_{1}} .
$$

The Mellin transform $M_{x}(s)$ of the T.F.N., where $x$ is positive, is as follows:

$$
\begin{aligned}
M_{x}(s)= & h_{p} \int_{a_{1}}^{a_{2}} x^{s-1}\left(\frac{x-a_{1}}{a_{2}-a_{1}}\right) d x \\
& +h_{p} \int_{a_{2}}^{a_{3}} x^{s-1}\left(\frac{x-a_{3}}{a_{2}-a_{3}}\right) d x, \\
= & h_{p}\left(\int_{a_{1}}^{a_{2}} x^{s-1}\left(\frac{x-a_{1}}{a_{2}-a_{1}}\right) d x\right. \\
& \left.+\int_{a_{2}}^{a_{3}} x^{s-1}\left(\frac{x-a_{3}}{a_{2}-a_{3}}\right) d x\right),
\end{aligned}
$$

$$
\begin{gathered}
=\left(\frac{h_{p}}{s(1+s)}\right) \\
\times\left(\left(\frac{a_{2}^{s} a_{1}(1+s)-\left(a_{2}^{1+s} s+a_{1}^{1+s}\right)}{\left(a_{1}-a_{2}\right)}\right)\right. \\
\left.+\left(\frac{a_{2}^{s} a_{3}(1+s)-\left(a_{3}^{1+s}+a_{2}^{1+s} s\right)}{\left(a_{2}-a_{3}\right)}\right)\right), \\
\quad\left(\frac{2(1+s)\left(a_{3}-a_{1}\right)}{s\left(\frac{a_{2}^{s} a_{1}(1+s)-\left(a_{2}^{1+s} s+a_{1}^{1+s}\right)}{\left(a_{1}-a_{2}\right)}\right)}\right) \\
\left.\quad\left(\frac{a_{2}^{s} a_{3}(1+s)-\left(a_{3}^{1+s}+a_{2}^{1+s} s\right)}{\left(a_{2}-a_{3}\right)}\right)\right) .
\end{gathered}
$$

To find the variance, we proceed as follows:

$$
\begin{aligned}
M_{x}(2)= & \left(\frac{2}{2(1+2)\left(a_{3}-a_{1}\right)}\right) \\
& \times\left(\left(\frac{a_{2}^{2} a_{1}(1+2)-\left(a_{2}^{1+2} 2+a_{1}^{1+2}\right)}{\left(a_{1}-a_{2}\right)}\right)\right. \\
& \left.+\left(\frac{a_{2}^{2} a_{3}(1+2)-\left(a_{3}^{1+2}+a_{2}^{1+2} 2\right)}{\left(a_{2}-a_{3}\right)}\right)\right), \\
M_{x}(3)= & \left(\frac{\left(a_{1}+a_{2}+a_{3}\right)}{3(1+3)\left(a_{3}-a_{1}\right)}\right) \\
& \times\left(\frac{a_{2}^{3} a_{1}(1+3)-\left(a_{2}^{1+3} 3+a_{1}^{1+3}\right)}{\left(a_{1}-a_{2}\right)}\right) \\
= & \frac{\left(a_{2}^{2}+a_{2} a_{1}+a_{3} a_{2}+a_{1}^{2}+a_{3} a_{1}+a_{3}^{2}\right)}{6} ; \\
& +\left(\frac{a_{2}^{3} a_{3}(1+3)-\left(a_{3}^{1+3}+a_{2}^{1+3} 3\right)}{\left(a_{2}-a_{3}\right)}\right),
\end{aligned}
$$

thus,

$$
\begin{aligned}
\operatorname{Var}(x)= & M_{x}(3)-\left(M_{x}(2)\right)^{2} \\
= & \frac{\left(a_{2}^{2}+a_{2} a_{1}+a_{3} a_{2}+a_{1}^{2}+a_{3} a_{1}+a_{3}^{2}\right)}{6} \\
& -\left(\frac{a_{1}+a_{2}+a_{3}}{3}\right)^{2}, \\
= & \frac{\left(a_{1}^{2}+a_{2}^{2}+a_{3}^{2}-a_{2} a_{1}-a_{3} a_{2}-a_{3} a_{1}\right)}{18} .
\end{aligned}
$$


To compute the Skewness and Kurtosis, we have

$$
\begin{aligned}
M_{x}(4)= & \left(\frac{2}{4(1+4)\left(a_{3}-a_{1}\right)}\right) \\
& \times\left(\left(\frac{a_{2}^{4} a_{1}(1+4)-\left(a_{2}^{1+4} 4+a_{1}^{1+4}\right)}{\left(a_{1}-a_{2}\right)}\right)\right. \\
& \left.+\left(\frac{a_{2}^{4} a_{3}(1+4)-\left(a_{3}^{1+4}+a_{2}^{1+4} 4\right)}{\left(a_{2}-a_{3}\right)}\right)\right), \\
= & \left(\left(a_{1}^{4}+a_{1}^{3} a_{2}+a_{1}^{2} a_{2}^{2}+a_{1} a_{2}^{3}-4 a_{2}^{4}+4 a_{2}^{4}\right)\right. \\
& \left.\quad-a_{3} a_{2}^{3}-a_{3}^{2} a_{2}^{2}-a_{2} a_{3}^{3}-a_{3}^{4}\right) \\
& \left.\times\left(10\left(a_{1}-a_{3}\right)\right)^{-1}\right), \\
M_{x}(5)= & \left(\left(a_{1}^{5}+a_{2} a_{1}^{4}+a_{2}^{2} a_{1}^{3}+a_{2}^{3} a_{1}^{2}+a_{1} a_{2}^{4}\right.\right. \\
& \left.\quad-a_{2}^{4} a_{3}-a_{3}^{2} a_{2}^{3}-a_{3}^{3} a_{2}^{2}-a_{3}^{4} a_{2}-a_{3}^{5}\right) \\
& \left.\times\left(15\left(a_{1}-a_{3}\right)\right)^{-1}\right) .
\end{aligned}
$$

Thus,

$$
\begin{aligned}
E(x-\mu)^{4}= & M_{x}(5)-4 M_{x}(2) M_{x}(4) \\
& +6\left(M_{x}(2)\right)^{2} M_{x}(3)-3\left(M_{x}(2)\right)^{4} \\
= & \left(\left(a_{1}^{4}-2 a_{1}^{3} a_{2}-2 a_{1}^{3} a_{3}+a_{3}^{4}-2 a_{2} a_{3}^{3}-2 a_{3} a_{2}^{3}\right.\right. \\
& \left.-2 a_{1} a_{3}^{3}-2 a_{1} a_{2}^{3}+a_{2}^{4}\right) \\
& \left.\times(135)^{-1}\right) \\
+ & \frac{\left(a_{1}^{2} a_{3}^{2}+a_{1}^{2} a_{2}^{2}+a_{3}^{2} a_{2}^{2}\right)}{45}
\end{aligned}
$$

and the kurtosis is given by

Kurtosis $(x)$

$$
\begin{aligned}
& =\frac{E(x-\mu)^{4}}{(\operatorname{Var}(x))^{2}} \\
& =\left(\left(\left(a_{1}^{4}-2 a_{1}^{3} a_{2}-2 a_{1}^{3} a_{3}+a_{3}^{4}-2 a_{2} a_{3}^{3}-2 a_{3} a_{2}^{3}\right.\right.\right. \\
& \left.\quad-2 a_{1} a_{3}^{3}-2 a_{1} a_{2}^{3}+a_{2}^{4}\right) \\
& \left.\quad \times(135)^{-1}\right) \\
& \left.+\left(\frac{\left(a_{1}^{2} a_{3}^{2}+a_{1}^{2} a_{2}^{2}+a_{3}^{2} a_{2}^{2}\right)}{45}\right)\right)
\end{aligned}
$$

$$
\begin{aligned}
& \times\left(\left(\frac{\left(a_{1}^{2}+a_{2}^{2}+a_{3}^{2}-a_{2} a_{1}-a_{3} a_{2}-a_{3} a_{1}\right)}{18}\right)^{2}\right)^{-1} \\
= & \frac{12}{5} .
\end{aligned}
$$

To find the skewness, we will need

$$
\begin{aligned}
E(x-\mu)^{3} & \\
= & M_{x}(4)-3 M_{x}(2) M_{x}(3)+2\left(M_{x}(2)\right)^{3} \\
= & \frac{\left(a_{1}^{3}+a_{2}^{3}+a_{3}^{3}\right)}{135} \\
& -\frac{\left(a_{1}^{2} a_{3}+a_{1}^{2} a_{2}+a_{1} a_{2}^{2}+a_{1} a_{3}^{2}+a_{3}^{2} a_{2}+a_{3} a_{2}^{2}\right)}{90} \\
& \left.+\frac{2 a_{1} a_{3} a_{2}}{45}\right) \\
= & \frac{\left(-a_{2}+2 a_{1}-a_{3}\right)\left(-2 a_{2}+a_{1}+a_{3}\right)\left(a_{2}+a_{1}-2 a_{3}\right)}{270} .
\end{aligned}
$$

Thus,

Skewness $(x)$

$$
\begin{aligned}
= & \left(\frac{\left(a_{1}^{3}+a_{2}^{3}+a_{3}^{3}\right)}{135}\right. \\
& -\frac{\left(a_{1}^{2} a_{3}+a_{1}^{2} a_{2}+a_{1} a_{2}^{2}+a_{1} a_{3}^{2}+a_{3}^{2} a_{2}+a_{3} a_{2}^{2}\right)}{90} \\
& \left.+\frac{2 a_{1} a_{3} a_{2}}{45}\right)
\end{aligned}
$$

$$
\times\left(\left(\sqrt{\frac{\left(a_{1}^{2}+a_{2}^{2}+a_{3}^{2}-a_{2} a_{1}-a_{3} a_{2}-a_{3} a_{1}\right)}{18}}\right)^{3}\right)^{-1}
$$

$$
\begin{aligned}
=( & \left(4\left(a_{1}^{3}+a_{2}^{3}+a_{3}^{3}\right)\right. \\
& -6\left(a_{1}^{2} a_{3}+a_{1}^{2} a_{2}+a_{1} a_{2}^{2}+a_{1} a_{3}^{2}+a_{3}^{2} a_{2}+a_{3} a_{2}^{2}\right) \\
& \left.+24 a_{1} a_{3} a_{2}\right) \\
\times & \left(5\left(a_{1}^{2}+a_{2}^{2}+a_{3}^{2}-a_{2} a_{1}-a_{3} a_{2}-a_{3} a_{1}\right)\right. \\
& \left.\left.\times \sqrt{\left(2 a_{1}^{2}+2 a_{2}^{2}+2 a_{3}^{2}-2 a_{2} a_{1}-2 a_{3} a_{2}-2 a_{3} a_{1}\right)}\right)^{-1}\right) .
\end{aligned}
$$


Remark 10. It is important to note here that, for the case of a Tr.F.N., we have the following easy to prove results:

$$
\begin{aligned}
& M_{x}(s)=\left(\frac{2}{\left(a_{4}+a_{3}\right)-\left(a_{2}+a_{1}\right)}\right) \\
& \times\left(\frac{a_{2}^{s} a_{1}+a_{2}^{s} a_{1} s-a_{2}^{1+s} s-a_{1}^{1+s}}{\left(a_{1}-a_{2}\right) s(1+s)}\right. \\
&+\frac{a_{3}^{s}-a_{2}^{s}}{s} \\
&\left.-\frac{a_{3}^{s} a_{4}-a_{4}^{1+s}+a_{3}^{s} s a_{4}-a_{3}^{1+s} s}{\left(a_{4}-a_{3}\right) s(1+s)}\right), \\
& M_{x}(2)=\frac{1}{3}\left(\frac{a_{3}^{2}+a_{4}^{2}+a_{4} a_{3}-a_{1}^{2}-a_{2} a_{1}-a_{2}^{2}}{a_{4}+a_{3}-a_{2}-a_{1}}\right),
\end{aligned}
$$

$M_{x}(3)$

$$
\begin{aligned}
& =\frac{1}{6}\left(\frac{a_{3}^{3}+a_{4}^{3}+a_{3} a_{4}^{2}+a_{4} a_{3}^{2}-a_{1}^{3}-a_{1}^{2} a_{2}-a_{1} a_{2}^{2}-a_{2}^{3}}{a_{4}+a_{3}-a_{2}-a_{1}}\right), \\
& M_{x}(4)=\frac{1}{10} \\
& \times\left(\left(a_{3}^{4}+a_{4}^{4}+a_{3} a_{4}^{3}+a_{3}^{2} a_{4}^{2}+a_{4} a_{3}^{3}-a_{1}^{4}\right.\right. \\
& \left.-a_{1}^{3} a_{2}-a_{1}^{2} a_{2}^{2}-a_{1} a_{2}^{3}-a_{2}^{4}\right) \\
& \left.\times\left(a_{4}+a_{3}-a_{2}-a_{1}\right)^{-1}\right), \\
& M_{x}(5)=\frac{1}{15} \\
& \times\left(\left(-a_{1}^{5}-a_{2} a_{1}^{4}-a_{2}^{2} a_{1}^{3}-a_{2}^{3} a_{1}^{2}-a_{1} a_{2}^{4}-a_{2}^{5}\right.\right. \\
& \left.+a_{3}^{5}+a_{4}^{5}+a_{3} a_{4}^{4}+a_{3}^{2} a_{4}^{3}+a_{3}^{3} a_{4}^{2}+a_{4} a_{3}^{4}\right) \\
& \left.\times\left(a_{4}+a_{3}-a_{2}-a_{1}\right)^{-1}\right),
\end{aligned}
$$

$\operatorname{Var}(x)$

$$
\begin{aligned}
= & \frac{1}{6}\left(\frac{a_{3}^{3}+a_{4}^{3}+a_{3} a_{4}^{2}+a_{4} a_{3}^{2}-a_{1}^{3}-a_{1}^{2} a_{2}-a_{1} a_{2}^{2}-a_{2}^{3}}{a_{4}+a_{3}-a_{2}-a_{1}}\right) \\
& -\frac{1}{9}\left(\frac{\left(a_{3}^{2}+a_{4}^{2}+a_{4} a_{3}-a_{1}^{2}-a_{2} a_{1}-a_{2}^{2}\right)^{2}}{\left(a_{4}+a_{3}-a_{2}-a_{1}\right)^{2}}\right) .
\end{aligned}
$$

From the previous results, we can easily compute the skewness and kurtosis of $x$.

Example 11. Consider the following symmetric quadratic fuzzy number given by

$$
\mu(x)=1-\left(\frac{x-a}{a}\right)^{2} \text { if } 0 \leq x \leq 2 a .
$$

Then, we have the following easy to prove results (see Figure 1):

$$
\begin{aligned}
h_{p}=\frac{3}{4 a}, \quad & M_{x}(s)=3\left(\frac{2^{s} a^{s-1}}{(1+s)(s+2)}\right), \\
\operatorname{Var}(X)= & M_{x}(3)-\left(M_{x}(2)\right)^{2} \\
= & \left(3\left(\frac{2^{3} a^{3-1}}{(1+3)(3+2)}\right)\right) \\
& -\left(3\left(\frac{2^{2} a^{2-1}}{(1+2)(2+2)}\right)\right)^{2} \\
= & \frac{a^{2}}{5}
\end{aligned}
$$

$$
\begin{aligned}
\operatorname{Kurtosis}(x) & =\frac{E(x-\mu)^{4}}{(\operatorname{Var}(x))^{2}} \\
& =\frac{(3 / 35) a^{4}}{\left(a^{2} / 5\right)^{2}} \\
& =2.1429,
\end{aligned}
$$

Skewness $(x)$

$$
\begin{aligned}
& =\frac{(3 / 4 a) \int_{0}^{2 a}(x-a)^{4-1}\left(1-((x-a) / a)^{2}\right) d x}{\left(\sqrt{a^{2} / 5}\right)^{3}} \\
& =0 .
\end{aligned}
$$

3.1. Moment Generating Function. If random variable $X$ has a m.g.f., then the $r$ th order moment of its distribution is equal to the value of the $r$ th order derivative of its m.g.f. for $t=0$. In what follows, we will discuss the mgf of a T.F.N. and Tr.F.N and discuss some of theirs properties:

$$
\begin{aligned}
M_{X}(t)= & E\left(e^{t x}\right)=\int_{-\infty}^{\infty} e^{t x} f(x) d x \\
= & \frac{2}{a_{3}-a_{1}} \int_{a_{1}}^{a_{2}} e^{t x}\left(\frac{x-a_{1}}{a_{2}-a_{1}}\right) d x \\
& +\frac{2}{a_{3}-a_{1}} \int_{a_{2}}^{a_{3}} e^{t x}\left(\frac{x-a_{3}}{a_{2}-a_{3}}\right) d x \\
= & \frac{2}{a_{3}-a_{1}}\left(\frac{-e^{a_{2} t} a_{2} t+e^{a_{2} t}+e^{a_{2} t} a_{1} t-e^{a_{1} t}}{t^{2}\left(-a_{2}+a_{1}\right)}\right) \\
& -\frac{2}{a_{3}-a_{1}}\left(\frac{e^{a_{3} t}+e^{a_{2} t} a_{2} t-e^{a_{2} t}-e^{a_{2} t} a_{3} t}{t^{2}\left(a_{2}-a_{3}\right)}\right) .
\end{aligned}
$$




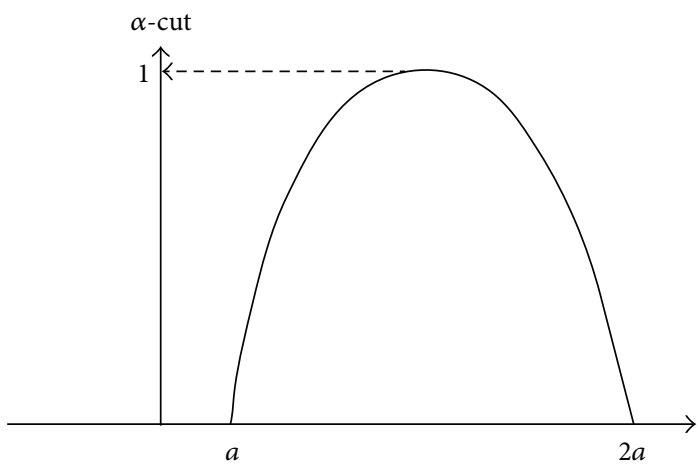

FIgURE 1: Nonlinear quadratic membership function.

The $r$ th moments can easily be computed as

$$
\begin{aligned}
& E\left(x^{r}\right)=\left.\frac{d^{r}}{d t^{r}} M_{X}(t)\right|_{t=0} \\
& =\left(\frac{2}{a_{3}-a_{1}}\right) \frac{d^{r}}{d t^{r}} \\
& \times\left[\left(\frac{-e^{a_{2} t} a_{2} t+e^{a_{2} t}+e^{a_{2} t} a_{1} t-e^{a_{1} t}}{t^{2}\left(-a_{2}+a_{1}\right)}\right)\right. \\
& \left.-\left(\frac{e^{a_{3} t}+e^{a_{2} t} a_{2} t-e^{a_{2} t}-e^{a_{2} t} a_{3} t}{t^{2}\left(a_{2}-a_{3}\right)}\right)\right]\left.\right|_{t=0} \\
& =\left(\frac{2}{a_{3}-a_{1}}\right) \\
& \times \lim _{t \rightarrow 0} \frac{d^{r}}{d t^{r}}\left[\left(\frac{-e^{a_{2} t} a_{2} t+e^{a_{2} t}+e^{a_{2} t} a_{1} t-e^{a_{1} t}}{t^{2}\left(-a_{2}+a_{1}\right)}\right)\right. \\
& \left.-\left(\frac{e^{a_{3} t}+e^{a_{2} t} a_{2} t-e^{a_{2} t}-e^{a_{2} t} a_{3} t}{t^{2}\left(a_{2}-a_{3}\right)}\right)\right] .
\end{aligned}
$$

The expected value is given by

$$
\begin{gathered}
E(x)=\left.\frac{d}{d t} M_{X}(t)\right|_{t=0} \\
=\lim _{t \rightarrow 0}\left(-2\left(\left(-e^{a_{2} t} a_{2}^{2} t^{2}+e^{a_{2} t} a_{2} a_{1} t^{2}\right.\right.\right. \\
\quad-e^{a_{2} t} a_{1} t-t a_{1} e^{a_{1} t} \\
\left.\quad+2 e^{a_{2} t} a_{2} t-2 e^{a_{2} t}+2 e^{a_{1} t}\right) \\
\left.\left.\times\left(\left(-a_{3}+a_{1}\right) t^{3}\left(-a_{2}+a_{1}\right)\right)^{-1}\right)\right) \\
+\lim _{t \rightarrow 0}\left(2 \left(\left(e^{a_{3} t} t a_{3}+e^{a_{2} t} a_{2}^{2} t^{2}\right.\right.\right. \\
\quad-e^{a_{2} t} a_{2} a_{3} t^{2}+e^{a_{2} t} a_{3} t-2 e^{a_{3} t} \\
\left.-2 e^{a_{2} t} a_{2} t+2 e^{a_{2} t}\right)
\end{gathered}
$$

$$
\begin{aligned}
& \left.\left.\times\left(\left(-a_{3}+a_{1}\right) t^{3}\left(a_{2}-a_{3}\right)\right)^{-1}\right)\right) \\
= & \left(\frac{1}{3} \frac{a_{1}^{2}+a_{2} a_{1}-2 a_{2}^{2}}{-a_{3}+a_{1}}\right) \\
& +\left(\frac{1}{3} \frac{2 a_{2}^{2}-a_{3} a_{2}-a_{3}^{2}}{-a_{3}+a_{1}}\right) \\
= & \frac{\left(a_{1}+a_{2}+a_{3}\right)}{3} .
\end{aligned}
$$

We can easily define the centered moment around the mean $\mu$ is

$$
\begin{aligned}
M_{X}(t) & =E\left(e^{t(x-\mu)}\right)=\int_{-\infty}^{\infty} e^{t(x-\mu)} f(x) d x \\
& =e^{-t \mu} \int_{-\infty}^{\infty} e^{t x} f(x) d x .
\end{aligned}
$$

Thus, we have

$$
\begin{aligned}
M_{X}^{C}(t)= & \frac{2}{a_{3}-a_{1}} e^{-t \mu} \int_{a_{1}}^{a_{2}} e^{t x}\left(\frac{x-a_{1}}{a_{2}-a_{1}}\right) d x \\
& +\frac{2}{a_{3}-a_{1}} e^{-t \mu} \int_{a_{2}}^{a_{3}} e^{t x}\left(\frac{x-a_{3}}{a_{2}-a_{3}}\right) d x \\
= & e^{-t \mu}\left(\frac{2}{a_{3}-a_{1}}\left(\frac{-e^{a_{2} t} a_{2} t+e^{a_{2} t}+e^{a_{2} t} a_{1} t-e^{a_{1} t}}{t^{2}\left(-a_{2}+a_{1}\right)}\right)\right. \\
& \left.-\frac{2}{a_{3}-a_{1}}\left(\frac{e^{a_{3} t}+e^{a_{2} t} a_{2} t-e^{a_{2} t}-e^{a_{2} t} a_{3} t}{t^{2}\left(a_{2}-a_{3}\right)}\right)\right)
\end{aligned}
$$

and the variance is

$$
\begin{aligned}
& \operatorname{Var}(x) \\
& =\lim _{t \rightarrow 0} \frac{d^{2}}{d t^{2}} \\
& \times\left(e^{-t \mu}\left(\begin{array}{c}
\frac{2}{a_{3}-a_{1}}\left(\frac{-e^{a_{2} t} a_{2} t+e^{a_{2} t}+e^{a_{2} t} a_{1} t-e^{a_{1} t}}{t^{2}\left(-a_{2}+a_{1}\right)}\right) \\
-\frac{2}{a_{3}-a_{1}}\left(\frac{e^{a_{3} t}+e^{a_{2} t} a_{2} t-e^{a_{2} t}-e^{a_{2} t} a_{3} t}{t^{2}\left(a_{2}-a_{3}\right)}\right)
\end{array}\right)\right) ;
\end{aligned}
$$


thus, we have $\operatorname{Var}(x)$, once we substitute for value of $\mu$ :

$\operatorname{Var}(x)$

$$
\begin{gathered}
=\frac{1}{6}\left(\left(a_{1}^{3}-4 \mu a_{1}^{2}+a_{1}^{2} a_{2}+6 \mu^{2} a_{1}-4 \mu a_{2} a_{1}\right.\right. \\
\left.+a_{1} a_{2}^{2}-3 a_{2}^{3}+8 \mu a_{2}^{2}-6 \mu^{2} a_{2}\right) \\
\left.\times\left(a_{1}-a_{3}\right)^{-1}\right) \\
+\frac{1}{6}\left(\left(3 a_{2}^{3}-a_{3} a_{2}^{2}-8 \mu a_{2}^{2}+6 \mu^{2} a_{2}+4 \mu a_{2} a_{3}\right.\right. \\
\left.-a_{3}^{2} a_{2}-6 \mu^{2} a_{3}+4 \mu a_{3}^{2}-a_{3}^{3}\right) \\
\left.\times\left(a_{1}-a_{3}\right)^{-1}\right) \\
=\frac{a_{1}^{2}-a_{2} a_{1}-a_{3} a_{1}+a_{2}^{2}-a_{3} a_{2}+a_{3}^{2}}{18} .
\end{gathered}
$$

Along similar lines we can also find the skewness and kurtosis as well.

Remark 12. The centered moments of a Tr.F.N is given by the following formula:

$$
\begin{aligned}
M_{X}(t)= & \left(2 e ^ { - t \mu } \left(\int_{a_{1}}^{a_{2}} e^{t x}\left(\frac{x-a_{1}}{a_{2}-a_{1}}\right) d x+\int_{a_{2}}^{a_{3}} e^{t x} d x\right.\right. \\
& \left.\left.+\int_{a_{3}}^{a_{4}} e^{t x}\left(\frac{x-a_{4}}{a_{3}-a_{4}}\right) d x\right)\right) \\
& \times\left(\left(a_{4}+a_{3}\right)-\left(a_{2}+a_{1}\right)\right)^{-1} .
\end{aligned}
$$

\section{Autoregressive Time Series Models}

Theorem 13. Consider the following AR(1) Models, where $y_{t}=\phi y_{t-1}+\varepsilon_{t}, \varepsilon_{t} \sim\left(M_{\varepsilon}(2), \sqrt{M_{\varepsilon}(3)-M_{\varepsilon}(2)}\right)$. Under the stationary conditions, $|\phi|<1$, we have the following moments in a fuzzy sense:

$$
\begin{aligned}
& E\left(y_{t}^{3}\right)=\left(6 M_{\varepsilon}^{2}(2) \phi^{3}+3 \phi\left(\phi-2 \phi^{2}+1\right) M_{\varepsilon}(2) M_{\varepsilon}(3)\right. \\
& \left.+M_{\varepsilon}(4)\left(1-\phi^{2}-\phi+\phi^{3}\right)\right) \\
& \times\left((1-\phi)\left(1-\phi^{2}\right)\left(1-\phi^{3}\right)\right)^{-1}, \\
& E\left(y_{t}^{4}\right) \\
& =\left(4 \phi ^ { 3 } \left(\left(6 M_{\varepsilon}^{2}(2) \phi^{3}+3 \phi\left(\phi-2 \phi^{2}+1\right) M_{\varepsilon}(2) M_{\varepsilon}(3)\right.\right.\right. \\
& \left.+M_{\varepsilon}(4)\left(1-\phi^{2}-\phi+\phi^{3}\right)\right) \\
& \left.\left.\times\left((1-\phi)\left(1-\phi^{2}\right)\left(1-\phi^{3}\right)\right)^{-1}\right) M_{\varepsilon}(2)\right) \\
& \times\left(\left(1-\phi^{4}\right)\right)^{-1} \\
& +\left(6 \phi^{2}\left(\frac{2 \phi M_{\varepsilon}^{2}(2)+M_{\varepsilon}(3)-\phi M_{\varepsilon}(3)}{(1-\phi)\left(1-\phi^{2}\right)}\right) M_{\varepsilon}(3)\right. \\
& \left.+4 \phi\left(\frac{M_{\varepsilon}(2)}{1-\phi}\right) M_{\varepsilon}(4)+M_{\varepsilon}(5)\right) \\
& \times\left(\left(1-\phi^{4}\right)\right)^{-1}
\end{aligned}
$$

Proof. Consider

$$
\begin{gathered}
E\left(y_{t}\right)=E\left(\phi y_{t-1}+\varepsilon_{t}\right)=\phi E\left(y_{t-1}\right)+M_{\varepsilon}(2), \\
E\left(y_{t}\right)-\phi E\left(y_{t-1}\right)=M_{\varepsilon}(2), \\
E\left(y_{t}\right)(1-\phi)=M_{\varepsilon}(2), \\
E\left(y_{t}\right)=\frac{M_{\varepsilon}(2)}{(1-\phi)} .
\end{gathered}
$$

The variance is computed as follows:

$$
\begin{aligned}
E\left(y_{t}^{2}\right) & =\frac{2 \phi E\left(y_{t-1}\right) E\left(\varepsilon_{t}\right)+E\left(\varepsilon_{t}^{2}\right)}{\left(1-\phi^{2}\right)} \\
& =\frac{2 \phi\left(M_{\varepsilon}(2) /(1-\phi)\right) M_{\varepsilon}(2)+M_{\varepsilon}(3)}{\left(1-\phi^{2}\right)} \\
& =\frac{2 \phi M_{\varepsilon}^{2}(2)+M_{\varepsilon}(3)-\phi M_{\varepsilon}(3)}{(1-\phi)\left(1-\phi^{2}\right)}
\end{aligned}
$$


thus,

$$
\begin{aligned}
\operatorname{Var}\left(y_{t}\right)= & \left(\frac{2 \phi M_{\varepsilon}^{2}(2)+M_{\varepsilon}(3)-\phi M_{\varepsilon}(3)}{(1-\phi)\left(1-\phi^{2}\right)}\right) \\
& -\left(\frac{M_{\varepsilon}(2)}{(1-\phi)}\right)^{2} \\
= & \frac{M_{\varepsilon}(3)-M_{\varepsilon}^{2}(2)}{1-\phi^{2}}, \\
E\left(y_{t}^{3}\right) & \\
= & \left(3 \phi^{2} E\left(y_{t-1}^{2}\right) E\left(\varepsilon_{t}\right)\right. \\
& \left.+3 \phi E\left(y_{t-1}\right) E\left(\varepsilon_{t}^{2}\right)+E\left(\varepsilon_{t}^{3}\right)\right) \\
& \times\left(\left(1-\phi^{3}\right)\right)^{-1} \\
= & \left(3 \phi^{2}\left(\frac{2 \phi M_{\varepsilon}^{2}(2)+M_{\varepsilon}(3)-\phi M_{\varepsilon}(3)}{(1-\phi)\left(1-\phi^{2}\right)}\right)\right. \\
& \quad \times M_{\varepsilon}(2) \\
& \left.+3 \phi\left(\frac{M_{\varepsilon}(2)}{1-\phi}\right) M_{\varepsilon}(3)+M_{\varepsilon}(4)\right) \\
& \times\left(\left(1-\phi^{3}\right)\right)^{-1} \\
= & \left(6 M_{\varepsilon}^{2}(2) \phi^{3}\right. \\
& +3 \phi\left(\phi-2 \phi^{2}+1\right) M_{\varepsilon}(2) M_{\varepsilon}(3) \\
& \left.+M_{\varepsilon}(4)\left(1-\phi^{2}-\phi+\phi^{3}\right)\right) \\
& \left.\left(1-\phi^{2}\right)\left(1-\phi^{3}\right)\right)^{-1}
\end{aligned}
$$

and the fourth moment is

$$
\begin{aligned}
& E\left(y_{t}^{4}\right) \\
& =\left(4 \phi^{3} E\left(y_{t-1}^{3}\right) M_{\varepsilon}(2)\right. \\
& \quad+6 \phi^{2}\left(\frac{2 \phi M_{\varepsilon}^{2}(2)+M_{\varepsilon}(3)-\phi M_{\varepsilon}(3)}{(1-\phi)\left(1-\phi^{2}\right)}\right) M_{\varepsilon}(3) \\
& \left.\quad+4 \phi\left(\frac{M_{\varepsilon}(2)}{1-\phi}\right) M_{\varepsilon}(4)+M_{\varepsilon}(5)\right) \\
& \quad \times\left(\left(1-\phi^{4}\right)\right)^{-1} \\
& =\left(4 \phi ^ { 3 } \left(\left(6 M_{\varepsilon}^{2}(2) \phi^{3}+3 \phi\left(\phi-2 \phi^{2}+1\right) M_{\varepsilon}(2) M_{\varepsilon}(3)\right.\right.\right. \\
& \left.\quad+M_{\varepsilon}(4)\left(1-\phi^{2}-\phi+\phi^{3}\right)\right)
\end{aligned}
$$

$$
\begin{aligned}
& \left.\left.\times\left((1-\phi)\left(1-\phi^{2}\right)\left(1-\phi^{3}\right)\right)^{-1}\right) M_{\varepsilon}(2)\right) \\
& \times\left(\left(1-\phi^{4}\right)\right)^{-1} \\
& +\left(6 \phi^{2}\left(\frac{2 \phi M_{\varepsilon}^{2}(2)+M_{\varepsilon}(3)-\phi M_{\varepsilon}(3)}{(1-\phi)\left(1-\phi^{2}\right)}\right) M_{\varepsilon}(3)\right. \\
& \left.\quad+4 \phi\left(\frac{M_{\varepsilon}(2)}{1-\phi}\right) M_{\varepsilon}(4)+M_{\varepsilon}(5)\right) \\
& \times\left(\left(1-\phi^{4}\right)\right)^{-1} .
\end{aligned}
$$

The skewness and kurtosis of the process follow from expressions for $\operatorname{Var}\left(y_{t}\right), E\left(y_{t}^{3}\right)$ and $E\left(y_{t}^{4}\right)$, respectively, and are omitted.

Remark 14. In Theorem 9, if we assume that the error term can be model by a Tr.F.N., given by $\varepsilon_{t}=[a, 2 a, 3 a, 4 a]$, then we can easily establish the following results:

$$
M_{\varepsilon}(s)=a^{s}\left(\frac{1-2^{1+s}-3^{1+s}+4^{1+s}}{2 a s(1+s)}\right) \text {, }
$$

where $M_{\varepsilon}(2)=(5 / 2) a, M_{\varepsilon}(3)=(20 / 3) a^{2}, M_{\varepsilon}(4)=(75 / 4) a^{3}$, $M_{\varepsilon}(5)=(826 / 15) a^{4}$, and $M_{\varepsilon}(6)=(335 / 2) a^{5}$ :

$$
\begin{gathered}
E\left(y_{t}\right)=\frac{5}{2}\left(\frac{a}{1-\phi}\right), \\
E\left(y_{t}^{2}\right)=\frac{5(7 \phi+8) a^{2}}{6(1-\phi)\left(1-\phi^{2}\right)}, \\
\operatorname{Var}(x)=\frac{5 a^{2}}{12\left(1-\phi^{2}\right)},
\end{gathered}
$$

$$
\begin{aligned}
& E\left(y_{t}^{3}\right) \\
& =\left(2 5 a ^ { 2 } \left(6 \phi^{3}+20 \phi^{2} a^{2}-40 a^{2} \phi^{3}+20 \phi a^{2}\right.\right. \\
& \left.\left.+3 a-3 a \phi^{2}-3 a \phi+3 a \phi^{3}\right)\right) \\
& \times\left(4(1-\phi)\left(1-\phi^{2}\right)\left(1-\phi^{3}\right)\right)^{-1}, \\
& E\left(y_{t}^{4}\right) \quad \\
& =\left(a ^ { 3 } \left(11250 \phi^{6}+3027 \phi^{5} a-27402 \phi^{6} a\right.\right. \\
& +5402 \phi^{4} a+7000 \phi^{3} a+6348 \phi^{2} a \\
& +3973 \phi a+1652 a)) \\
& \times\left(30(1-\phi)\left(1-\phi^{2}\right)\right. \\
& \left.\times\left(1-\phi^{3}\right)\left(1-\phi^{4}\right)\right)^{-1},
\end{aligned}
$$




$$
\begin{aligned}
& K^{(y)} \\
& =\left(2 4 \left(11250 \phi^{6}+3027 \phi^{5} a-27402 \phi^{6} a\right.\right. \\
& +5402 \phi^{4} a+7000 \phi^{3} a+6348 \phi^{2} a \\
& +3973 \phi a+1652 a)) \\
& \times\left(125\left(1-\phi^{3}+\phi^{2}-\phi\right)\right. \\
& \left.\times\left(1-\phi^{3}\right) a\right)^{-1}
\end{aligned}
$$

Skewness $\left(y_{t}\right)$

$$
\begin{aligned}
=((6 \sqrt{5} \sqrt{3}(\phi+1) \\
\times\left(-6 \phi^{3}-20 \phi^{2} a^{2}+40 a^{2} \phi^{3}\right. \\
-20 \phi a^{2}-3 a+3 a \phi^{2} \\
\left.\left.+3 a \phi-3 a \phi^{3}\right)\right) \\
\times\left(\sqrt{\left(1-\phi^{2}\right)}(1-\phi)\right. \\
\left.\left.\times\left(\phi^{2}+\phi+1\right) a\right)^{-1}\right) .
\end{aligned}
$$

4.1. Random Coefficient Volatility Models. Consider the class of random coefficient autoregressive (RCA) models defined by allowing random additive perturbations of the autoregressive (AR) coefficients of ordinary AR models. That is, we assume that the process $y_{t}$ is given by

$$
y_{t}-\sum_{i=1}^{p}\left(\phi_{i}+b_{i}(t)\right) y_{t-i}=e_{t},
$$

where the parameters $\theta_{i},(i=2, \ldots, p)$, are assumed to be known, $e_{t}$ and $b_{i}(t)$ are zero mean square integrable independent processes, and the variances are denoted by $\sigma_{e}^{2}$ and $\sigma_{b}^{2} \cdot b_{i}(t),(i=1,2, \ldots)^{\prime} s$ are independent of $e_{t}$ and $y_{t-i}$ and may be thought of as incorporating structural changes. In order to motivate nonlinear forecasts for nonlinear models, we consider a class of estimating functions of the form $g_{n}=$ $\sum_{i=2}^{n} b_{t-i} h_{t}$ as in Thavaneswaran and Heyde [9], where $h_{t}=$ $y_{t}-E\left[y_{t} \mid F_{t-1}^{y}\right]=y_{t}-\sum_{i=1}^{p} \phi_{i} y_{t-i}$ and $b_{t-i}$ is a function of $y_{1}, y_{2}, \ldots, y_{t-i}$ and possibly the known parameters $\phi_{1}, \ldots, \phi_{p}$ (i.e., We assume that the fitted model is available). If we restrict ourselves to a class of estimating functions of the previous form, then we can forecast the future value of $y_{n+1}$ based on the observed values $y_{1}, y_{2}, \ldots, y_{n}$ as $\widehat{y}_{n}(1)=$ $E\left[y_{n+1} \mid y_{n}, y_{n-1}, \ldots\right]$. That is, whether we have an $\operatorname{AR}(\mathrm{p})$ model or RCA(p) model, we will get the same linear predictor of $y_{n+1}$. However, for the RCA model under consideration, we have $E\left[\begin{array}{lll}y_{t} & \mid & F_{t-1}^{y}\end{array}\right]=\sum_{i=1}^{p} \phi_{i} y_{t-i}$ and $\operatorname{Var}\left[y_{t} F_{t-1}^{y}\right]=$ $\sigma_{\varepsilon}^{2}+\sum_{i=1}^{p} y_{t-i}^{2} \sigma_{b}^{2}$. Thus, the conditional variance is a nonlinear function and hence the RCA model may be viewed as a nonlinear time series model. Nicholls and Quinn [10] studied linear as well as some nonlinear (proposed) forecast by fitting a nonlinear (RCA) model for the classical lynx cycle data. Using heuristic reasoning, they proposed a nonlinear forecast $\widehat{y}_{n+1}=\operatorname{sgn}\left(\phi_{1} \widehat{y}_{n}\right)\left[\begin{array}{ll}\phi_{1}^{2} & \widehat{y}_{n}^{2}+\sigma_{\varepsilon}^{2}\end{array}\right]^{1 / 2}$ and they showed empirically that the forecast $\widehat{y}_{n+1}$ is a better predictor (having smaller forecast errors when compared with the actual observations) than the linear forecast for the lynx data. It is of interest to note that, by defining $h_{t}=y_{t}^{2}-E\left[\begin{array}{l|l}y_{t}^{2} & F_{t-1}^{y}\end{array}\right]$, the optimal forecast for $y_{n+1}$ can be obtained as $y_{n}^{*}(1)=$ $\left[E\left[y_{t}^{2} \mid F_{t-1}^{y}\right]\right]^{1 / 2}=\operatorname{sgn}\left(\phi y_{n}^{2}\right)\left[\psi_{1}^{2}+\sigma_{n}^{2}+\sigma_{\varepsilon}^{2}\right]^{1 / 2}$. That is, the estimating function method can be used to obtain a nonlinear forecast for a nonlinear models by considering a class of elementary martingale estimating functions generated by nonlinear functions of the observations. Using a similar argument, we could also obtain forecasts for various classes of GARCH models; see Thavaneswaran and Heyde [9] for details. The main message is that RCA models could be used to improve the forecasting performance of stochastic volatility models. Appadoo et al. [11] derive the kurtosis of the correlated RCA model as well as the normal GARCH model under the assumption that the errors are correlated. Appadoo et al. [12] consider some volatility models with quadratic $\mathrm{GARCH}$ innovations and derive the kurtosis of the process.

4.2. RCA Models. Random coefficient autoregressive time series were introduced by Nicholls and Quinn [10] and some of their properties have been studied recently by Thavaneswaran et al. [13]. RCA models exhibiting long memory properties have been considered in Leipus and Sugailis [14]. A sequence of random variables $\left\{y_{t}\right\}$ is called an RCA(1) time series if it satisfies the equations

$$
y_{t}=\left(\phi+b_{t}\right) y_{t-1}+\varepsilon_{t} \quad t \in Z,
$$

where $Z$ denotes the set of integers and

$$
\begin{aligned}
& \text { (i) }\left(\begin{array}{l}
b_{t} \\
\varepsilon_{t}
\end{array}\right) \sim\left(\left(\begin{array}{l}
0 \\
0
\end{array}\right),\left(\begin{array}{cc}
\sigma_{b}^{2} & 0 \\
0 & \sigma_{\varepsilon}^{2}
\end{array}\right)\right), \\
& \text { (ii) } \phi^{2}+\sigma_{b}^{2}<1 .
\end{aligned}
$$

The sequences $\left\{b_{t}\right\}$ and $\left\{\varepsilon_{t}\right\}$, respectively, are the errors in the model.

Theorem 15. Let $\left\{y_{t}\right\}$ be an RCA(1) time series satisfying as in conditions ( $i)$ and (ii), and let $\gamma_{y}(k)$ be its covariance function. Then, one has the following.

(a) $E y_{t}=0, E y_{t}^{2}=\sigma_{e}^{2} /\left(1-\phi^{2}-\sigma_{b}^{2}\right)$, the kth lag autocovariance for $y_{t}$ is given by $\gamma_{y}(k)=\phi^{k} \sigma_{e}^{2} /\left(1-\phi^{2}-\right.$ $\sigma_{b}^{2}$ ) and the autocorrelation for $y_{t}$ is $\rho_{k}=\phi^{k}$ for all $k \in Z$. That is, the usual AR(1) process has same autocorrelation as the RCA(1).

(b) If $\left\{b_{t}\right\}$ and $\left\{e_{t}\right\}$ are normally distributed random variables, then the unconditional kurtosis $K^{(y)}$ of the RCA process $\left\{y_{t}\right\}$ is given by

$$
K^{(y)}=\frac{3\left[1-\left(\sigma_{b}^{2}+\phi^{2}\right)^{2}\right]}{\left[1-\left(\phi^{4}+6 \phi^{2} \sigma_{b}^{2}+3 \sigma_{b}^{4}\right)\right]}
$$

and for an $A R(1)$ process $K^{(y)}$ reduces to 3. 
(c) The autocorrelation of $y_{t}^{2}$ is given by $\rho_{k}^{y^{2}}=\left(\phi^{2}+\sigma_{b}^{2}\right)^{k}$ and for an $A R(1)$ process it turns out to be $\rho_{k}^{y^{2}}=\phi^{2 k}$.

Corollary 16. Let $\left\{y_{t}\right\}$ be an RCA(1) time series of the form $y_{t}=\theta_{t} y_{t-1}+e_{t}$ satisfying the stationarity conditions, and let $\rho_{k}^{y}$ denote its correlation function. Then, one has the following:

(a) when $\theta_{t}=\phi+b_{t}, E y_{t}=0, E y_{t}^{2}=\sigma_{e}^{2} /\left(1-\phi^{2}-\sigma_{b}^{2}\right)$, the kth lag autocorrelation for $y_{t}$ is given by $\rho_{k}^{y}=\{E(\phi+$ $\left.\left.b_{t}\right)\right\}^{k}=\phi^{k}$

(b) when $\theta_{t}=\operatorname{sgn}\left(b_{t}\right)$ where $b_{t} \sim N\left(0, \sigma_{b}^{2}\right)$, then $\rho_{k}^{y}=$ $[1-2 F(0)]^{k}$, where $F$ is the cumulative distribution function of $b_{t}$ (i.e) when the coefficient $\theta_{t}$ is driven by a binary random variable $\left\{b_{t}\right\}$ taking values -1 and +1 .

(c) when $\theta_{t}=\left(\phi+\left|b_{t}\right|^{\alpha}\right)$ where $b_{t} \sim N\left(\mu, \sigma_{b}^{2}\right)$ then, the autocorrelation

$$
\rho_{k}^{y}=\left[\phi+\frac{\left(2 \sigma_{b}^{2}\right)^{\alpha / 2}}{\sqrt{\pi}} \Gamma\left(\frac{\alpha+1}{2}\right)\right]^{k}
$$

where $\Gamma(\cdot)$ is the Gamma function.

Theorem 17. Consider the following RCA(1) Models, where $y_{t}=\left(\phi+b_{t}\right) y_{t-1}+\varepsilon_{t}$, and $b_{t} \sim N\left(0, \sigma_{b}^{2}\right), \varepsilon_{t} \sim\left(a,(6 / 5) a^{2}\right)$ and $\varepsilon_{t} \sim 1-((x-a) / a)^{2}$, for $0 \leq x \leq 2 a$. Under the stationary conditions, $|\phi|<1$ then one has the following moments in a fuzzy sense:

$$
\begin{gathered}
E\left(y_{t}\right)=\frac{a}{(1-\phi)} \\
E\left(y_{t}^{2}\right)=\frac{2 a^{2}(2 \phi+3)}{5(1-\phi)\left(1-\phi^{2}-\sigma_{b}^{2}\right)} \\
\operatorname{Var}\left(y_{t}\right)=\frac{\left(2 \phi-\phi^{2}-1-5 \sigma_{b}^{2}\right) a^{2}}{5(1-\phi)^{2}\left(1-\phi^{2}-\sigma_{b}^{2}\right)} \\
E\left(y_{t}^{3}\right)=\frac{2 a^{3}\left(\phi^{3}+5 \phi^{2}+5 \phi+\phi \sigma_{b}^{2}+5 \sigma_{b}^{2}+4\right)}{5(1-\phi)\left(1-\phi^{2}-\sigma_{b}^{2}\right)\left(1-\phi^{3}+3 \phi \sigma_{b}^{2}\right)}
\end{gathered}
$$

Skewness $\left(y_{t}\right)$

$$
\begin{aligned}
& =\frac{2 a^{3}\left(\phi^{3}+5 \phi^{2}+5 \phi+\phi \sigma_{b}^{2}+5 \sigma_{b}^{2}+4\right)}{5(1-\phi)\left(1-\phi^{2}-\sigma_{b}^{2}\right)\left(1-\phi^{3}+3 \phi \sigma_{b}^{2}\right)} \\
& \times\left(\left(\sqrt{\frac{\left(2 \phi-\phi^{2}-1-5 \sigma_{b}^{2}\right) a^{2}}{5(1-\phi)^{2}\left(1-\phi^{2}-\sigma_{b}^{2}\right)}}\right)^{3}\right)^{-1},
\end{aligned}
$$

$$
\begin{aligned}
& E\left(y_{t}^{4}\right) \\
& =\left(8 a ^ { 4 } \left(606 \phi \sigma_{b}^{2}+139 \sigma_{b}^{2}+978 \phi^{3} \sigma_{b}^{2}\right.\right. \\
& +50+85 \phi^{4}+795 \phi^{2} \sigma_{b}^{2} \\
& +213 \phi^{2} \sigma_{b}^{4}+942 \sigma_{b}^{4} \phi+139 \phi^{2} \\
& +126 \phi^{3}+90 \phi-\phi^{6}+36 \phi^{5} \\
& \left.\left.+212 \phi^{4} \sigma_{b}^{2}\right)\right) \\
& \times\left(175(1-\phi)\left(1-\phi^{2}-\sigma_{b}^{2}\right)\right. \\
& \times\left(1-\phi^{3}+3 \phi \sigma_{b}^{2}\right) \\
& \left.\times\left(1-6 \phi^{2} \sigma_{b}^{2}-\phi^{4}-4 \sigma_{b}^{3}\right)\right)^{-1} .
\end{aligned}
$$

The kurtosis of the process is

$$
\begin{aligned}
& K^{(y)} \\
&=\left(8 a^{4}\left(5(1-\phi)^{2}\left(1-\phi^{2}-\sigma_{b}^{2}\right)\right)^{2}\right. \\
& \times\left(606 \phi \sigma_{b}^{2}+139 \sigma_{b}^{2}+978 \phi^{3} \sigma_{b}^{2}\right. \\
&+50+85 \phi^{4}+795 \phi^{2} \sigma_{b}^{2}+213 \phi^{2} \sigma_{b}^{4} \\
&+942 \sigma_{b}^{4} \phi+139 \phi^{2}+126 \phi^{3} \\
&\left.\left.+90 \phi-\phi^{6}+36 \phi^{5}+212 \phi^{4} \sigma_{b}^{2}\right)\right) \\
& \times\left(175(1-\phi)\left(1-\phi^{2}-\sigma_{b}^{2}\right)\right. \\
& \times\left(1-\phi^{3}+3 \phi \sigma_{b}^{2}\right) \\
& \times\left(1-6 \phi^{2} \sigma_{b}^{2}-\phi^{4}-4 \sigma_{b}^{3}\right) \\
&\left.\times\left(\left(2 \phi-\phi^{2}-1-5 \sigma_{b}^{2}\right) a^{2}\right)^{2}\right)^{-1}
\end{aligned}
$$

\section{Conclusion}

The authors feel that the approach developed in this paper may be applicable in other areas as well. There are several applications of the Mellin transformation in statistical inference. For example, Samanta [15] used the Mellin transformation (through a modified Bessel function tabulated in Abramowitz and Stegun [16]) to obtain the minimum variance unbiased estimation (MVUE) of a probability function. The method is based on finding solution of a certain integral function. The results obtained using fuzzy assumptions in this paper are more flexible, and the methodology proposed in this paper may also be applicable for other time series models. The evidence in favor of the fuzzy approach supported by the Mellin transpose highlights the advantage of the model developed in this paper. 


\section{Conflict of Interests}

The authors declare that there is no conflict of interests.

\section{Acknowledgments}

The authors wish to thank two anonymous reviewers for their insightful comments and suggestions that have resulted in an improved version of the manuscript. The authors wish to acknowledge the financial support of this work by Discovery Grants from the Natural Sciences and Engineering Research Council (NSERC) of Canada.

\section{References}

[1] C. R. Bector and S. Chandra, Fuzzy Mathematical Programming and Fuzzy Matrix Games, vol. 169 of Studies in Fuzziness and Soft Computing, Springer, New York, NY, USA, 2005.

[2] A. Kaufmann and M. M. Gupta, Introduction to Fuzzy Arithmetic, Van Nostrand Reinhold Company, New York, NY, USA, 1985.

[3] J. N. Sheen, "Fuzzy financial profitability analyses of demand side management alternatives from participant perspective," Information Sciences, vol. 169, no. 3-4, pp. 329-364, 2005.

[4] A. Erdelyi, Table of Integral Transforms, vol. 1, McGraw-Hill, New York, NY, USA, 1954.

[5] G. Doetsch, Handbuch der Laplace Transformation, vol. 1, Birkhäuser, Basel, Switzerland, 1950.

[6] E. C. Titchmarsh, Introduction to the Theory of Fourier Integrals, Claredon Press, Oxford, UK, 1937.

[7] O. González-Gaxiola and J. A. Santiago, "An $\alpha$-Mellin transform and some of its applications," International Journal of Contemporary Mathematical Sciences, vol. 7, no. 45-48, pp. 2353-2361, 2012.

[8] R. Frontczak and R. Schöbel, "On modified Mellin transforms, Gauss-Laguerre quadrature, and the valuation of American call options," Journal of Computational and Applied Mathematics, vol. 234, no. 5, pp. 1559-1571, 2010.

[9] A. Thavaneswaran and C. C. Heyde, "Prediction via estimating functions," Journal of Statistical Planning and Inference, vol. 77, no. 1, pp. 89-101, 1999.

[10] D. F. Nicholls and B. G. Quinn, Random Coefficient Autoregressive Models: An Introduction, vol. 11 of Lecture Notes in Statistics, Springer, New York, NY, USA, 1982.

[11] S. S. Appadoo, A. Thavaneswaran, and J. Singh, "RCA models with correlated errors," Applied Mathematics Letters, vol. 19, no. 8, pp. 824-829, 2006.

[12] S. S. Appadoo, A. Thavaneswaran, and S. Mandal, "RCA model with quadratic GARCH innovation distribution," Applied Mathematics Letters, vol. 25, no. 10, pp. 1452-1457, 2012.

[13] A. Thavaneswaran, S. S. Appadoo, and M. Ghahramani, "RCA models with GARCH innovations," Applied Mathematics Letters, vol. 22, no. 1, pp. 110-114, 2009.

[14] R. Leipus and D. Surgailis, "Random coefficient autoregression, regime switching and long memory," Advances in Applied Probability, vol. 35, no. 3, pp. 737-754, 2003.

[15] M. Samanta, "A unified approach to minimum variance unbiased estimation of a probability function belonging to an exponential family," Communications in Statistics, vol. 17, no. 10, pp. 3413-3426, 1988.
[16] M. Abramowitz and I. A. Stegun, Handbook of Mathematical Functions, Dover, New York, NY, USA, 1970. 


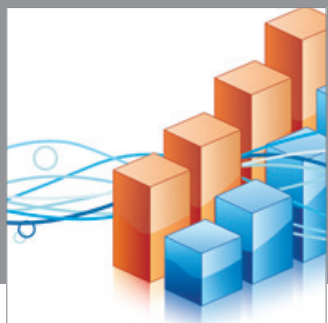

Advances in

Operations Research

mansans

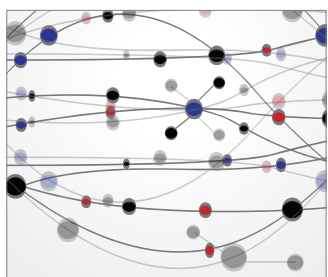

The Scientific World Journal
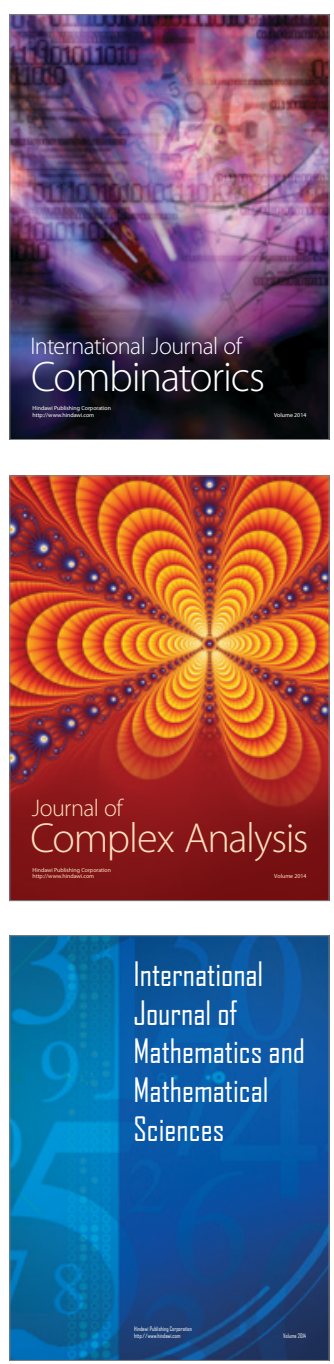
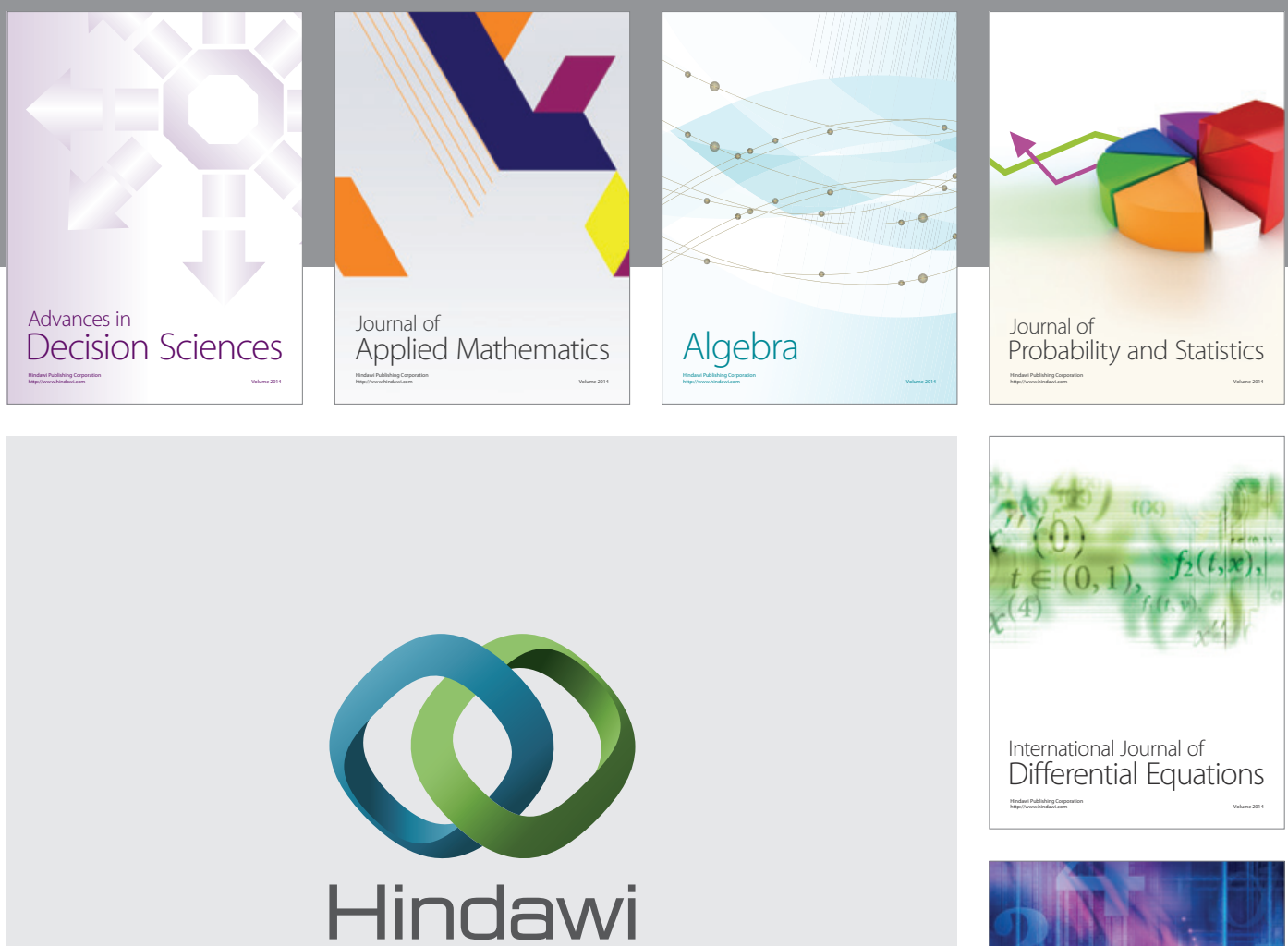

Submit your manuscripts at http://www.hindawi.com
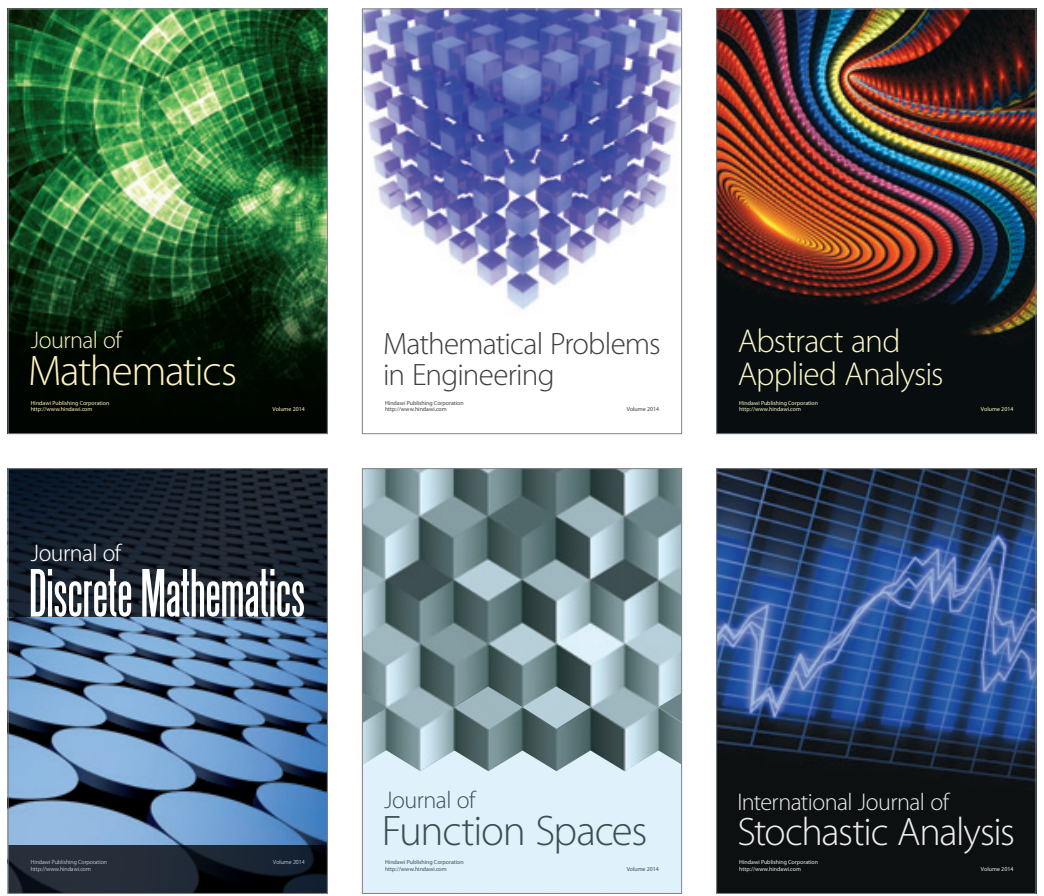

Journal of

Function Spaces

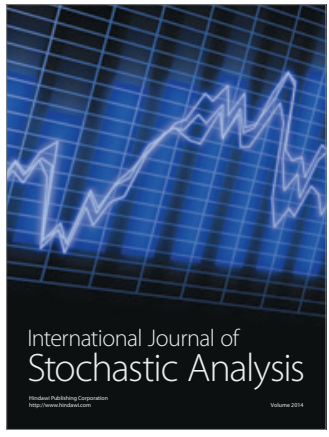

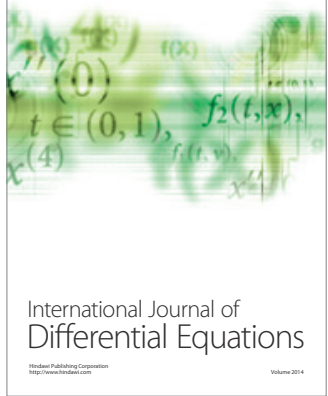
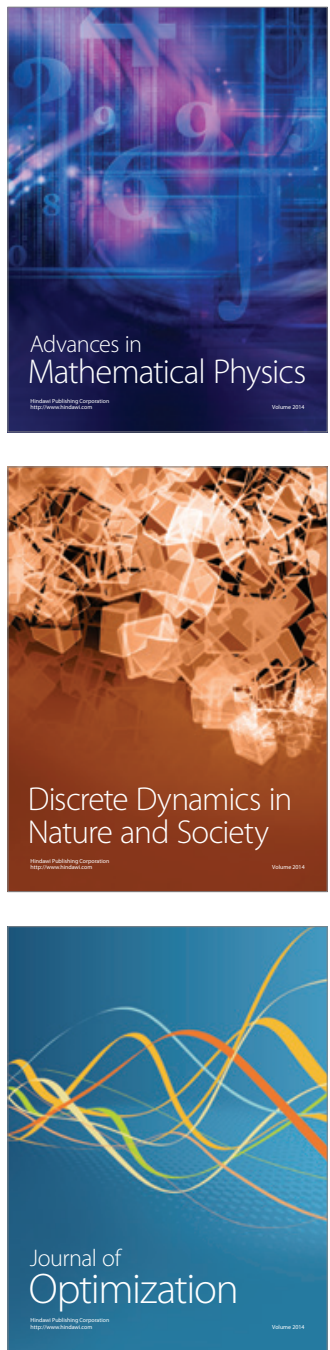